\title{
Listening with Curiosity - Tracking the Acoustic Response of Portable Laser Ablation ${ }^{*}$
}

\author{
Stefan Kradolfer ${ }^{\star \star a}$, Kurt Heutschib ${ }^{\mathrm{b}}$ Joachim Kocha, and Detlef Günthera \\ §SCS-DSM award for the best virtual poster in Analytical Sciences
}

\begin{abstract}
Nowadays, one of the methods of choice for minimal invasive sampling of solid matter is laser ablation (LA). Routine LA sampling is performed commonly in the laboratory and the amount of ablated mass can directly be monitored and analysed. By contrast laser-based sampling in the field, using a portable laser ablation system $(\mathrm{pLA})$, still remains challenging concerning low-absorbing or NIR-transparent samples. The current hardware is limited in regards to photon energy and density resulting in unsteady ablation. But as the actual amount of collected mass is the major crux of on-site sampling, with this performance it is often unknown and estimates can only be made based on the experience from prior method development and the experience of the user. In the following work an easy-to-use method to monitor the amount of ablated material collected during laser-based sampling by measuring the acoustic response is presented. The pLA-system was coupled to inductively coupled plasma mass spectrometry (ICPMS) via a diffusion driven gas exchange device (GED) which allowed to monitor mass removal and acoustic response quasi-simultaneously. For the current instrumentation only actual mass removal leads to the formation of shockwaves (SW) and, thus, acoustic signals. These events can be used as indicator for executed LA events and counted on an individual basis. The intensity of acoustic signals has been shown to correlate with the LA mass, i.e., the amount of ablated material. This allows to perform re-adjustment of the laser focus during sampling for optimal ablation based on the intensity of the acoustic signal. Likewise, acoustic intensity together with counting allows the operator to make estimates about total mass sampled. Therefore, unsuccessful laser aerosol collection in the field shall become a thing of the past.
\end{abstract}

Keywords: Acoustic tracking · Laser induced plasma · Portable laser ablation sampling · Sampling in the field

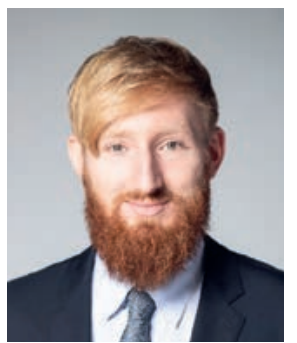

Stefan Kradolfer obtained his bachelor and master degrees in chemistry from ETH Zürich. During this time, he left ETH for an internship at the AVSV (Amt für Verbraucherschutz und Veterinärwesen) in the canton St. Gallen where he investigated the degradation products of minced meat by headspace analysis via GC-MS. He carried out his master thesis in the group of Prof. Renato Zenobi at ETH Zurich on the electron transfer properties of the blue copper-protein Azurin by tip-enhanced Raman spectroscopy. In 2016, he started his doctoral studies in the group of Prof. Detlef Günther at ETH Zurich with the focus on elemental analysis for archaeometric purposes using laser-based sampling.

"The research described in this article is published in Spectrochimica Acta Part B: Atomic Spectroscopy, S. Kradolfer, K. Heutschi, J. Koch and D. Günther, 'Tracking mass removal of portable laser ablation sampling by its acoustic response', https://doi.org/10.1016/j.sab.2021.106118. Copyright Elsevier (2021).

\section{Introduction}

Laser-based sampling of solid materials for analytical purposes is almost as old as the invention of the laser itself. ${ }^{[1-3]}$ The removal of substance through exposure to laser pulses is called laser ablation (LA), whereby the laser irradiance exceeds the material- and the wavelength-specific threshold value and the solid sample undergoes decomposition.

This decomposition is accompanied by the following processes: solid-to-liquid-to-vapour 'forward' phase transitions of sample material and the formation of aerosols through nucleation, condensation and agglomeration, ${ }^{[4]}$ including emergence and expansion of a laser induced plasma (LIP). ${ }^{[5]}$ Over the past few decades LA has been studied intensively and the interest is still growing based on its versatile applications.

LA as a microprobe sampling tool originates from high-resolution material processing. ${ }^{[6]}$ Over time, the wish of scientists and, in particular, (analytical) chemists for multi-element- and isotope-selective analyses of the ablated material led to the development of various LA-based 'hyphenated' techniques. These are various including direct mass spectrometry (LAMA/LAMS), ${ }^{[7,8]}$ LIP spectroscopy as laser induced breakdown spectroscopy LIBS, ${ }^{[5]}$ the operation with secondary ionization sources such as 
inductively-coupled plasmas (ICP's) for both optical emission spectroscopy OES ${ }^{[9]}$ or mass spectrometry MS. ${ }^{[10]}$

The linking of laser ablation with inductively coupled plasma mass spectrometry as LA-ICPMS is nowadays considered to be one of the methods of choice for the determination of the elemental/isotopic composition of solids, including their major, minor, trace and ultra-trace elements. LA-ICPMS is characterised by relatively simple handling of samples (no high vacuum required), high spatial resolution down to $\mu \mathrm{m}$-levels, wide linear-dynamic range up to nine orders of magnitude in combination with low limits of detection reaching attogramme concentrations. ${ }^{[11]}$ Nevertheless, LA-ICPMS requires a laboratory infrastructure with controlled and stable settings, including a well-defined ablation area. LA is most commonly accomplished using an airtight ablation cell flushed with inert gases such as argon or helium. This leads to restrictions in terms of sample size; typically up to a few centimetres. However, many samples especially in the field of archaeology or geology are either too big or bulky, immobile or not moveable due to regulatory restrictions and are consequently not accessible for laboratory based analysis.

Therefore, from 2010 on our research group conceptualized and developed a portable laser ablation system (pLA). ${ }^{[12-14]}$ With the aid of this instrument it has become possible to analyse the aforementioned objects by sampling on-site and measuring the elemental composition back in the laboratory. pLA combines quasi non-destructive sampling of conventional LA with the almost unlimited sampling capabilities in terms of sample size and site of a portable device. In comparison to other portable devices, such as pLIBS or pXRF, ${ }^{[15]}$ it enables analysis to nearly all metals and oxides of the periodic table plus isotopic information, while providing overall low limits of detection compared to the other techniques.

The pLA system was designed to be robust, lightweight and reliable. It consists of a Nd:YAG laser operated at a VIS wavelength of $532 \mathrm{~nm}$ connected to a handheld LA module via an optical fibre. The LA aerosols are drawn by deposition on filters using a membrane pump positioned downstream. Notably, the VIS laser wavelength used enables low-loss light transmission through the optical waveguides while offering sufficient laser pulse and photon energy to decompose even dielectric materials. In comparison, UV light guided in a similar way would suffer a substantial loss of energy on its way. The setup has been successfully used for analysis of metallic and NIR absorbing samples, ${ }^{[12,16,17]}$ but the drawbacks concerning pulse energy, photon energy and fluence do not allow sampling of low-absorbing or NIR-transparent samples, such as ceramics, glasses or certain minerals. No, weak, or unsteady LA leads to a lower ablated amount than required for further analysis, ${ }^{[18]}$ resulting in unsuccessful field trips.

During every LA-event up to half of the energy can be converted into an acoustic shockwave (SW), ${ }^{[19]}$ which can be heard by the human ear and recorded by a microphone. The SW has been shown to not only indicate $\mathrm{LA}^{[20]}$ but also to estimate the amount of mass removal. ${ }^{[21]}$ This allowed to use it for normalization purposes in the sense of internal standardization, e.g. correlation between acoustic and OES-signals has been reported. ${ }^{[20-25]}$ Ever since the beginning of acoustic tracking of LA, the high sample dependency proved to be a mayor disadvantage and prevented a broader application of this technique. Nevertheless, in recent years the concept has been revived,[26] especially in the field of laser induced breakdown spectroscopy (LIBS). ${ }^{[26-28]}$

\section{Experimental Approach for the Acoustic Tracking of Laser Ablation}

This work explores the concept using acoustic SWs created during LA sampling as marker and measure for mass removal. All experiments were performed by an in-house built portable LA system, as described by Glaus et al..$^{[12,14]}$ The handheld module was re-constructed and equipped with a capacitor microphone for SW acoustic monitoring, ${ }^{[12,14]}$ (BY-M1, BOYA, Shenzhen Jiayz Photo Industrial ;audio interface: scarlett 2i4, focusrite, High Wycombe, GB, see Fig. 1). An acoustic channel was integrated at the lower end of the module, which together with the micro-

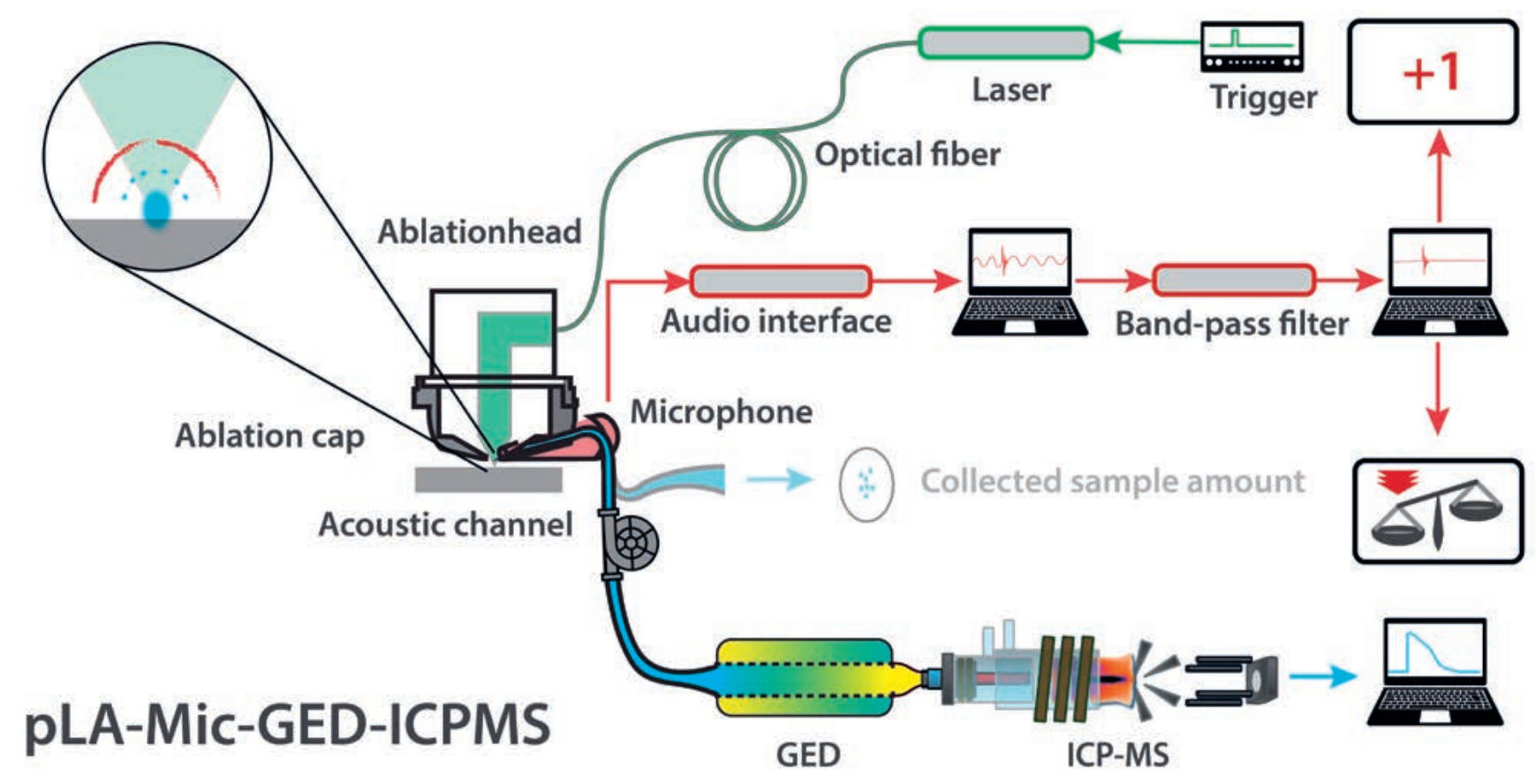

Fig. 1. Setup for the investigation of the acoustic signal produced by pLA-sampling. The laser source is triggered resulting in a laser ablation pulse delivered by a glass fibre to the ablation head of the pLA-System. The laser is focused on the sample leading to mass removal due to absorption of the laser on the sample surface of the solid. As the fast expansion shockwave detaches, it is tracked by the microphone (Mic) which is coupled to an acoustic channel in the ablation cap (red track). With the information of the acoustic data, one is able to count each laser ablation event including mass removal $(+1)$ and allows to estimate the total ablated amount of material. The ablated mass can either be collected in the field for subsequent analysis in the laboratory or directly analysed by the connection to an ICPMS via a diffusion driven gas exchange device (GED, air-to-argon). 
phone served as directional microphone. This allows to narrow the focus of the measurement to the LA site. By monitoring the acoustic pressure, it became possible to count every successful LA event and, thus, to estimate the amount of material removed. Background noise and vibrations from the lab setting (and especially from the membrane pump responsible for the transport of the ablated mass) turned out to interfere with the LA acoustic signals in their low-frequency band and reduced the microphone sensitivity. The acoustic signal was therefore pre-processed prior to counting using a FFT band-stop filter for covering frequencies between approximately 100 and $1500 \mathrm{~Hz}$, which enabled to substantially suppress background noise while only minimally compromising the actual signal.

Calibration of the directional microphone allowed to measure absolute pressure readings (in units of $[\mathrm{Pa}]$ ) to the otherwise arbitrary amplitude values. Acoustic signals were evaluated using three different approaches with increasing information content:

1. Counting: The acoustic signal produced by LA is measured as a sudden deviation or discontinuity in the acoustic signature (peak), which results in a broadband increase in power spectral density. This can be distinguished from background signal and allows for counting actual mass removal events.

2. Sum of the maximal amplitudes: The maximal pressure deviation of the discontinuity is representing the main energy of the formed shockwave and serves as a more detailed description. This information allows to compare ablation events with each other. Either directly during the measurement where the relative signal intensity can be used for instant quality control of the laser focus, with the possibility for direct readjustment, or by the sum over a series of ablation events as indicator of its entity.

3. Event level: Taking into account the total signal structure, such as the oscillation over time, and variation in-between different signals, the Event Level (EL), as the integration over the squared signal, is an even more precise measure for the acoustic energy in a series of ablation events. However, the accumulation over a longer time period amplifies the effect of the background noise. This measure is commonly used for the description of intense, single acoustic events such as gun shots.

The comparison of acoustic signals and ablated mass was accomplished using the pLA-system in combination with ICPMS. For this purpose, ambient air was exchanged with Argon by a diffusion driven gas exchange device (GED, air-to-argon). ${ }^{[16]}$ In doing so the detour including time- and resource intensive wet chemistry were avoided. In ICPMS, while operating in their linear-dynamic range the signal is directly proportional to the introduced mass and is therefore directly useable as a measure of mass.

\subsection{Theoretical Aspects of LA Acoustic Monitoring}

According to definition, LA is the process of removing material from a solid's surface by exposure to (pulsed) laser radiation. This results in the formation of SW's whose intensity is assumed to relate to the amount of mass removed during LA. The aim of our study was to get a better understanding of the relation between the laser properties, as energy and ablation area, and the sample dependent ablation process. The specific interest was set in regards of the total amount of mass removal and the intensity of the formed acoustic shockwave.

$$
E_{S W}=C \cdot\left[E_{T}-\Delta E_{H D} \pm \sum_{i} \Delta E_{ \pm}^{i}\right]
$$

The model calculations are based on an energy conservation approach that is summarized by Eqn. (1). The shock wave energy $E_{\mathrm{SW}}$ is given by the remaining energy stemming from the total energy of the laser absorbed by the target, given as $E_{\mathrm{T}}$, minus losses due to heat diffusion $\left(\Delta E_{\mathrm{HD}}\right)$ plus the sum of additional contributions/deductions $\left(\Delta E^{\mathrm{i}}\right)$, as $e$.g. gain of energy due to plasma shielding or losses due to thermal radiation, times a correction factor $C$. The calculations depend highly on the laser- and material-parameter, which influence the different energy terms. The calculations were performed for the model system of pure copper and a ns-laser with a wavelength of $532 \mathrm{~nm}$, as used within the pLA-system. The model allowed varying the energy of the laser and the dimension of the ablation spot, while providing the absolute mass removal (in pg) and the amplitude pressure of the acoustic shockwave (in Pa). In Fig. 2, the results for a fixed laser energy $\left(E_{0}=0.5 \mathrm{~mJ}\right)$ and the variation of the spot size is shown. Changes in energy density during the ablation with the pLA are common as the energy can instrumentally be fixed but small variation in the positioning of the laser focus on the samples lead to changes in the spot-size, given by the short focal length of the setup.

The calculation reveals that the acoustic signal is directly related to the energy density: the higher the energy density, the higher the corresponding acoustic energy. This enables to use the acoustic energy as an indication for the quality of the laser focus, where the smallest spot-size is desired. On the other hand, the mass removal increases for an increasing spot-size, but reaches a maximum above which the amount is decreasing again. This max-
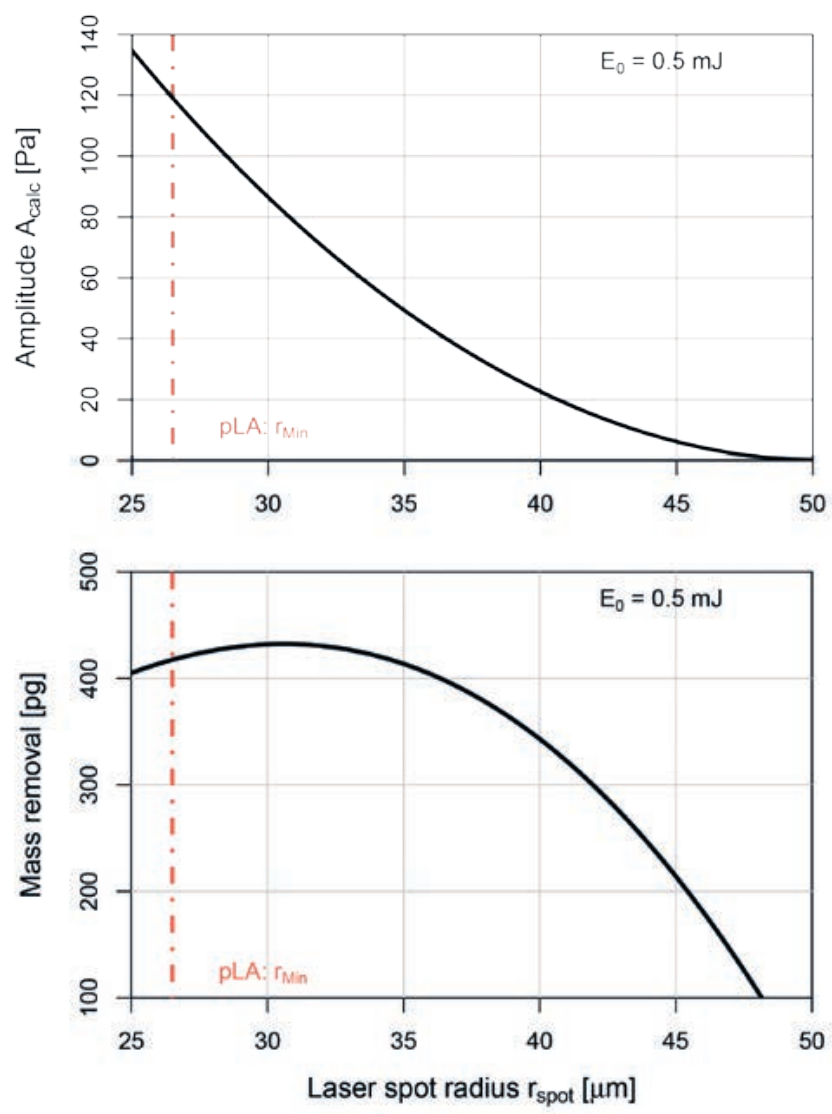

Fig. 2. The model calculation presented is based on an energy conservation approach given by Eqn. (1) for a pure copper sample and a ns-laser with $532 \mathrm{~nm}$ wavelength. The laser energy $E_{0}$ was set to $0.5 \mathrm{~mJ}$ while the spot size and therefore the fluence was changed ( $x$-axis). The red line indicates the minimal radius $r_{\min }$ on which the laser of the pLA-system can be focused. Top: With increasing spot-size the calculated acoustic amplitude decreases steadily until it reaches zero with a $r_{\text {spot }}$ of roughly $50 \mu \mathrm{m}$. Bottom: The mass removal [pg] increases with increasing spot-size as the penetration depth is limited and the energy is sufficient for full mass removal. Around $r_{\text {spot }} 30 \mu \mathrm{m}$ the ablated mass reaches a maximum and decreases with increasing spot-size as the amount of energy is not sufficient for complete mass removal. 
imal ablation is in the accessible region of the portable laser. A precise calibration for the mass removal is therefore challenging and can only be given with a high uncertainty.

\subsection{Acoustic Tracking of Mass Removal}

Performing experiments with the described setup including various samples showed consistency regarding the joint appearance of signal for the mass removal, given by an ICPMS response, and an acoustic signal. Acoustic signal due to a laser-induced air-breakdown was experimentally excluded beforehand. Samples with low absorbance regarding the laser wavelength of $532 \mathrm{~nm}$, e.g. calcite, showed no response on both channels. On the contrary, all acoustic signals could be linked to actual mass removal. Therefore, the acoustic signal is suitable to count all the actual LA-events leading to mass removal.

\subsection{Ablation of Metal Samples}

The pLA-system was initially designed for sampling materials with low ablation thresholds in the VIS spectral range, e.g. metals. The ablation threshold of these samples is about an order of magnitude lower than that required for non-metals and no problems concerning ablation were encountered. The investigation of single ablation events as part of sequence of pulses for hole drilling experiments showed an ambiguous picture (see Fig. 3). A possible explanation can be given by the theoretical calculation in section 2.1. The triangular spread is in agreement with the model calculation, as increasing acoustic signal amplitudes imply broader possible mass related responses. Nevertheless, the description of overall trends with the investigation into these samples was possible. As in conventional LA, the first few laser pulses in hole drilling experiments show different behaviour compared to the later ones, by high, apparent random variability on both channels. This can be explained by surface effects and different absorption behaviour resulting in a different response regarding mass removal and intensity of the acoustic pressure wave. As in conventional LA-ICPMS, the signal slowly decreases with progressing ablation process. The same was observed for the acoustic signal. This is in agreement with the expectation of the correlation between the two measures. In general, the acoustic tracking of the pLA-sampling allows to re-adjust the focal position of the laser on the sample, as the higher the signal the better in focus is the laser.

\subsection{Ablation of Low-absorbing Samples}

With a recent modification on the pLA-system, the fluence on the sample during ablation could be increased from $\sim 9 \mathrm{~J} / \mathrm{cm}^{2}$ up to $34 \mathrm{~J} / \mathrm{cm}^{2}{ }^{2}{ }^{[18]}$ This allows to access samples with a comparably high ablation threshold, such as glass, minerals or porcelain. Nevertheless, the newly reached energy density is on the limit for steady and clean ablation, with the consequence of possibly unsteady sampling. The samples for this study were the standard reference material NIST SRM 610 and the iron rich mineral BCR$2 \mathrm{G}$. Investigations with single ablation events turned out to be challenging, instead continuously possibly random ablation with a LA frequency of $10 \mathrm{~Hz}$ were executed. Hole drilling experiments from 20 up to 308 consecutive LA-events allowed to compare the different measurement characteristics. Advantageously with the average over a collection of many laser pulses reduced the influence of the first few pulses.

In every experiment, the number of pulses according the acoustic response, the sum over all maximal amplitudes per event and the event level were compared to the sum of the ICPMS response (see Fig. 4). A positive correlation was observed. A linear best fit was applied with an increasing accuracy for the latter two measures, given by an increase in $\mathrm{R}^{2}$. The lower spread in the data, compared to the samples with a comparably low ablation threshold, can be explained by the maximal reachable fluence on the LA-site, which is close to the ablation threshold of the samples.

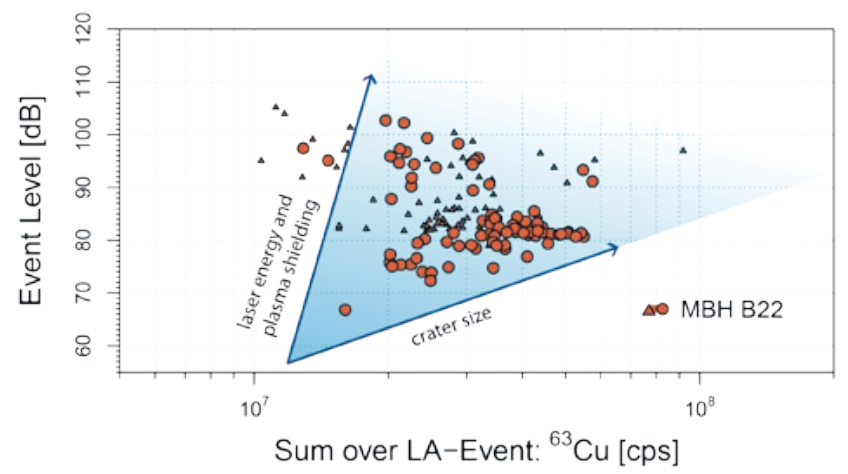

Fig. 3: Results of pLA-GED-ICPMS drilling experiments (1 up to 30 events) for the brass reference material MBH B22 (Cu: 82.47 wt\%, Zn: 15.92 wt\%). The sum of the ICPMS-response [cps] on ${ }^{63} \mathrm{Cu}$ for single pLA events on the $x$-axis is plotted against the measured Event Level $[\mathrm{dB}]$ on the $y$-axis. The first three LA events in the respective drilling experiments, given as triangular shaped data points, show deviations in signal intensity compared to latter events, as shown in conventional LAICPMS. These are therefore not taken into account for the data evaluation and discussion. The data is fanning out for increasing values of both axes. As the model calculation already showed, the trend of the data can be explained for the acoustic signal with its fluence dependency, mostly influenced by changes in laser energy accompanied by plasma shielding. For conducting metal samples, which are less fluence dependent for LA, an increase in crater size results in more mass removal.

The closer these values are, the smaller spread can be expected. This is in agreement with the theoretical model calculation, as seen in section 2.1.

The quality of the trend line allows to conclude that the acoustic measure is a valid option to describe the mass removal of the entity of consecutive LA pulses, for these specific samples. Sampling by pLA includes in any case a detailed method development whereas the sample amount is assured by several measures. The acoustic tracking presents itself as a relatively convenient way that can be included in the method development as being part of the quality assurance of the entire sampling procedure. The acquired acoustic data can be used for comparison during on-site sampling and can serve as a calibration.

\section{Conclusion}

Simultaneous measurements of the acoustic responses during pLA-sampling and the amount of mass removal by the coupling to GED-ICPMS have been developed and studied for both metallic and non-metallic samples. For the current instrumentation, the occurrence of acoustic responses is consistently accompanied by mass removal. Continuous recording allows to measure and count the LA events on an individual level. Data treatment, including a band-stop filter in the lower frequency range for background noise reduction and an external calibration, allows to assign an absolute pressure level $[\mathrm{Pa}]$ to every event and making a comparison possible.

Model calculations show the complex nature of the relation between the laser matter interaction, the ablation process including mass removal and the acoustic signal of the generated shockwave. The intensity of the acoustic signal can be used as a relative indicator for the laser focus position resulting in the smallest crater sizes, whereas the link to the mass is not strictly given. These findings are supported by single pulse experiments of pure copper and brass samples.

The presented approach provides the possibility to count every actual laser ablation event and allows qualitative information during (possibly unsteady) ablation in the field. Consecutive multi-pulse experiments, including 20 up to 308 of LA-events, show a linear response for the comparison of the acoustic signal and the mass re- 


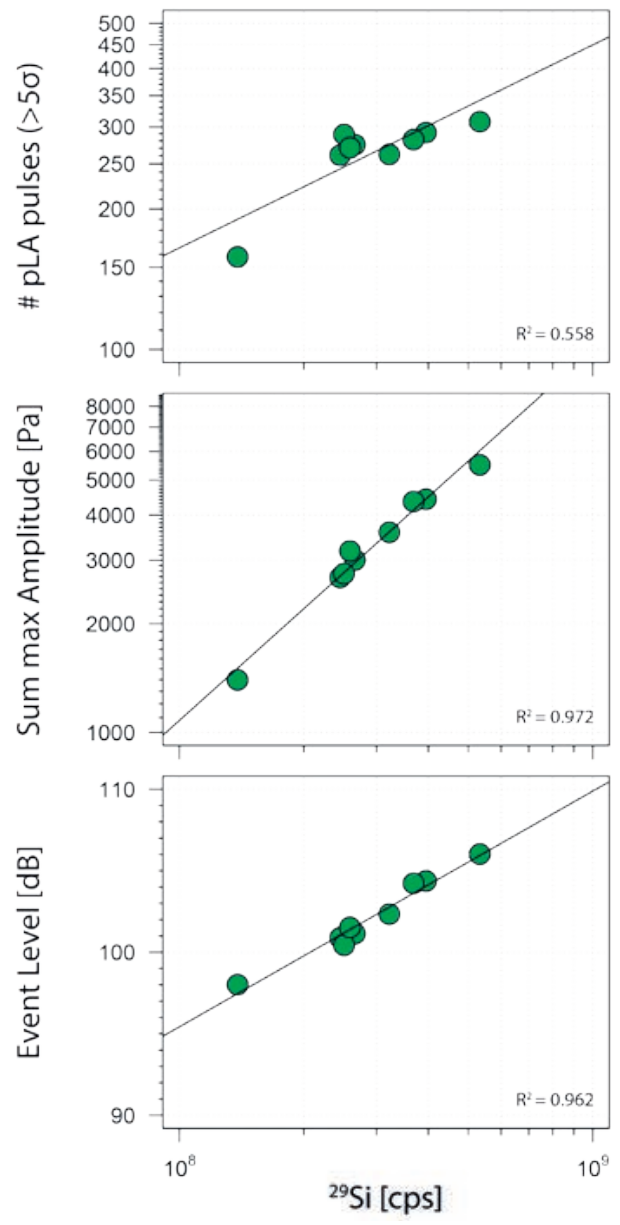

Fig. 4. The acoustic response of multi-pulse experiments with the iron rich mineral $\mathrm{BCR}-2 \mathrm{G}$ is presented: the number of counted events (top), the sum of the maximal amplitude over all counted events (middle) and the Event Level over the measurement session (bottom). These data were compared to the sum over the ICPMS response ( $x$-axis), with the example for the main element silicon measured on ${ }^{29} \mathrm{Si}$. A linear relation was assumed and a line of best fit was included, given with the corresponding $\mathrm{R}^{2}$ as a measure for accuracy.

moval of samples with a comparably high ablation threshold. The accuracy is higher for an indicator that uses the sum over the amplitude of the acoustic events or the event level (EL), in contrast to simply counting the events. Furthermore, in combination with a method development prior to the field work it allows for an educated estimation of the total mass removal. Insufficient sampling can immediately be confirmed and, if possible, actions can be taken. The portable laser ablation system presents itself as versatile tool for offline sampling, and in combination with the acoustic tracking it is even more reliable.

Acoustic tracking of the ablation event is one alternative to assure material collection outside the laboratory by pLA. However, another approach could be using the optical (or OES spectral) analysis of the LIP, as in LIBS, by implementing a light sensor or even a small spectrometer. Nevertheless, these approaches face the same problems as the current approach, as they are all material- and fluence-dependent. Another expansion in this direction would be feasible, but would also increase the complexity of the system and could restrict its portability.

\section{Acknowledgements}

This article is based on the article published in Spectrochimica Acta Part B: Atomic Spectroscopy as being part of a special issue in honour of the lifetime achievements of Prof. Dr. Richard E. Russo in spectrochemistry and especially in the field of LA fundamentals and LIBS. The financial (ETH Zurich) and infrastructure support (ETH Zurich, Empa Dübendorf), assistance through the mechanical workshop of D-CHAB, especially Roland Mäder and Roland Walker, and scientific input and discussion with Lorenzo Querci and Lyndsey Hendriks is gratefully acknowledged.

Received: January 29, 2021

[1] F. Brech, W. J. Young, in 'Application of Science in Examination of Works of Art. Proceedings of the Seminar: September 7-16, 1965, Conducted by the Research Laboratory, Museum of Fine Arts, Boston, Massachusetts', 1965, pp. 230.

[2] K. Niemax, in 'Optoelectronics for Environmental Science', Springer US, 1990, pp. 229, https://doi.org/10.1007/978-1-4684-5895-4_20.

[3] R. E. Russo, Appl. Spectrosc. 1995, 49, 14A.

[4] J. Koch, A. Von Bohlen, R. Hergenröder, K. Niemax, J. Anal. At. Spectrom. 2004, 19, 267, https://doi.org/10.1039/b310512a.

[5] F. Breech, L. Cross, Appl. Spectrosc. 1962, 16, 1.

[6] D. Bäuerle, 'Laser Processign and Chemistry', 2011, Springer, https://doi.org/10.1007/978-3-642-17613-5.

[7] N. C. Fenner, N. R. Daly, Rev. Sci. Instrum. 1966, 37, 1068, https://doi.org/10.1063/1.1720410.

[8] L. Querci, V. Varentsov, D. Günther, B. Hattendorf, Spectrochim. Acta B 2018, 146, 57, https://doi.org/10.1016/j.sab.2018.05.004.

[9] M. Thompson, J. E. Coulter, F. Sieper, Analyst 1981, 106, 32, https://doi.org/10.1039/AN9810600032.

[10] A. L. Gray, Analyst 1985, 110, 551, https://doi.org/10.1039/AN9851000551.

[11] D. Günther, B. Hattendorf, TrAC Trends Anal. Chem. 2005, 24, 255, https://doi.org/10.1016/j.trac.2004.11.017.

[12] R. Glaus, J. Koch, D. Günther, Anal. Chem. 2012, 84, 5358, https://doi.org/10.1021/ac3008626.

[13] R. Glaus, L. Dorta, Z. Zhang, Q. Ma, H. Berke, D. Günther, J. Anal. At. Spectrom. 2013, 28, 801, https://doi.org/10.1039/c3ja30379a.

[14] R. Glaus, $\mathrm{PhD}$ Thesis ETH Zürich, 2013, https://doi.org/10.3929/ethz-a-009906484.

[15] G. S. Senesi, R. S. Harmon, R. R. Hark, Spectrochim. Acta B 2021, 175, 106013, https://doi.org/10.1016/j.sab.2020.106013.

[16] M. Burger, R. Glaus, V. Hubert, S. van Willigen, M. Wörle-Soares, F. Convertini, P. Lefranc, E. Nielsen, D. Günther, J. Archaeol. Sci. 2017, 82, 62, https://doi.org/10.1016/j.jas.2017.04.009.

[17] A. C. S. Knaf, J. M. Koornneef, G. R. Davies, J. Anal. At. Spectrom. 2017 31, 2210, https://doi.org/10.1039/C7JA00191F.

[18] D. Käser, ETH MSc thesis, 'Redesign of a portable laser ablation setup to allow sampling of ancient Chinese Jade and Porcelain', 2015, https://doi.org/10.3929/ethz-b-000189964.

[19] J. Koch, S. Heiroth, T. Lippert, D. Günther, Spectrochim. Acta - Part B At. Spectrosc. 2010, 65, 943, https://doi.org/10.1016/j.sab.2010.09.004.

[20] J. Diaci, J. Mozina, Appl. Phys. A Solids Surf. 1992, 55, 352, https://doi.org/10.1007/BF00324084.

[21] G. Chen, E. S. Yeung, Anal. Chem. 1988, 60, 2258, https://doi.org/10.1021/ac00171a020.

[22] H.-M. Pang, E. S. Yeung, Anal. Chem. 1989, 61, 2546 , https://doi.org/10.1021/ac00197a018.

[23] H.-M. Pang, D. R. Wiederin, R. S. Houk, E. S. Yeung, Anal. Chem. 1991, 63, 390, https://doi.org/10.1021/ac00004a017.

[24] C. Stauter, P. Gérard, J. Fontaine, T. Engel, Appl. Surf. Sci. 1997, 109-110, 174, https://doi.org/10.1016/S0169-4332(96)00655-1.

[25] S. Palanco, J. Laserna, Appl. Opt. 2003, 42, 6078, https://doi.org/10.1364/ao.42.006078.

[26] B. Campanella, S. Legnaioli, S. Pagnotta, F. Poggialini, V. Palleschi, Atoms 2019, 7, 57, https://doi.org/10.3390/ATOMS7020057.

[27] B. Chide, S. Maurice, N. Murdoch, J. Lasue, B. Bousquet, X. Jacob, A Cousin, O. Forni, O. Gasnault, P. Y. Meslin, J. F. Fronton, M. Bassas-Portús, A. Cadu, A. Sournac, D. Mimoun, R. C. Wiens, Spectrochim. Acta - Part B At. Spectrosc. 2019, 153, 50, https://doi.org/10.1016/j.sab.2019.01.008.

[28] B. Chide, S. Maurice, A. Cousin, B. Bousquet, D. Mimoun, O. Beyssac, P.-Y. Meslin, R. C. Wiens, Spectrochim. Acta Part B At. Spectrosc. 2020, 106000, https://doi.org/10.1016/j.sab.2020.106000.

\section{License and Terms}

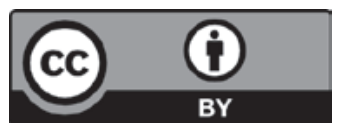

This is an Open Access article under the terms of the Creative Commons Attribution License CC BY 4.0. The material may not be used for commercial purposes.

The license is subject to the CHIMIA terms and conditions: (http:// chimia.ch/component/sppagebuilder/?view=page \&id=12).

The definitive version of this article is the electronic one that can be found at https://doi.org/10.2533/chimia.2021.300 